\title{
Fisher information, delocalization and the semiclassical description of molecular rotation
}

\author{
S Curilef ${ }^{1}$, F Pennini $^{1,2}$, A Plastino ${ }^{2}$ and G L Ferri ${ }^{3}$ \\ ${ }^{1}$ Departamento de Física, Universidad Católica del Norte, Av. Angamos 0610, \\ Antofagasta, Chile \\ ${ }^{2}$ Instituto de Física La Plata (CONICET-UNLP), Fac. de Ciencias Exactas, \\ Universidad Nacional de La Plata, CC 67, 1900 La Plata, Argentina \\ ${ }^{3}$ Facultad de Ciencias Exactas, Universidad Nacional de La Pampa, Peru y Uruguay, \\ Santa Rosa, La Pampa, Argentina
}

Received 3 January 2007, in final form 22 March 2007

Published 24 April 2007

Online at stacks.iop.org/JPhysA/40/5127

\begin{abstract}
We discuss phase-space delocalization for the rigid rotor within a semiclassical context by recourse to the Husimi distributions of both the linear and the 3Danisotropic instances. The Husimi function can be viewed in a variety of ways. In particular, we obtain it as the expectation value of the density operator in a suitable basis of coherent states. Our treatment is based upon the concomitant Fisher information measures. The pertinent Wehrl entropy is also investigated in the linear case.
\end{abstract}

PACS numbers: 03.67.-a, 05.30.-d, 31.15.Gy

\section{Introduction}

Using Fisher's information measure as our main tool, we will be concerned here with the semiclassical description of the rotational dynamics of molecular systems. A pioneer analysis of such rotational dynamics is that of Morales et al in [1], who studied the connection between it and coherent states [2, 3].

The coherent states (CS) formulation is not unique and several authors have developed alternative descriptions for molecular quantum systems. We need CS in this work because one uses them so as to construct Husimi semiclassical distributions (see sections 2.2 and 3.1). With the latter, we determine Fisher's measure (see section 3.2):

\section{Coherent states $\rightarrow$ Husimi distribution $\rightarrow$ Fisher's information measure.}

We have chosen two traditional formulations for coherent states in order to discuss the semiclassical dynamics of molecular rotational systems. One of them is devoted to twodimensional case [4] and the other to the three-dimensional one [1]. 
In $[5,6]$ the authors discuss, among other things, the advantages of the Husimi distribution for the interpretation of the electronic structure in hydrogen and nitrogen atoms. They suggested that their work may be extended to the molecular instance. Following this suggestion we address here the simplest applicable model, i.e., the rigid rotor. Its usefulness for describing diatomic molecules is well known [7].

Delocalization is an 'energetically favourable' process, since it distributes the wavefunction over a volume greater than the size of the sample. Thus, the net energy of the molecule is lowered, which results in resonance stabilization. The celebrated Fisher information $\mathcal{F}$ [9-12] can be related (for example by Garbaczewski [8]) to the inverse of the delocalization measure as follows: if we take a wave package with a standard deviation $\sigma$, the Fisher information is given by $\mathcal{F} \geqslant 1 / \sigma^{2}$, thus, in this sense, we realize that the Fisher information is a quite sensitive indicator of the delocalization of the wave package [9-11].

The rigid rotor is a system of a single particle whose quantum spectrum of energy is exactly known. Therefore, the study of typical thermodynamic properties can be analytically derived [13]. Applications lead to the treatment of important aspects of molecular systems [14] and several applications to materials [15-18].

The paper is organized as follows: in section 2, we introduce basic aspects and properties which must be satisfied by coherent states. In section 3, we explore the linear rigid rotor. We write the probability of finding a quantum state in a coherent state that is used to obtain an explicit expression for the (i) Husimi distribution, (ii) Wehrl entropy and (iii) Fisher Information. These results are of help in section 4, where we discuss a model for the threedimensional rigid rotor. Finally, in section 5, we draw some concluding remarks.

\section{Coherent states: harmonic oscillator}

Quantum-mechanical phase-space distributions expressed in terms of the coherent states $|z\rangle$ of the harmonic oscillator [2] have been proved to be useful in different contexts. Particular reference is made to the important work of Anderson and Halliwell [19], who discuss, among other things, the concepts of Husimi distribution and Wehrl entropy. Coherent states are eigenstates of the annihilation operator $\hat{a}$, appropriate for the problem at hand, i.e.,

$$
\hat{a}|z\rangle=z|z\rangle,
$$

whose Hamiltonian reads $\hat{H}_{o}=\hbar \omega\left[\hat{a}^{\dagger} \hat{a}+1 / 2\right]$ and the complex eigenvalues of the annihilation operator $\hat{a}$ are given by

$$
z=(m \omega / 2 \hbar)^{1 / 2} x+\mathrm{i}(2 \hbar \omega m)^{-1 / 2} p .
$$

\subsection{Requirements for a formulation of coherent states}

It is well known that coherent states can be constructed in several ways and by recourse to different techniques, its formulation being of a non-unique character. Gazeau and Klauder suggest that a suitable formalism for coherent states should satisfy the following criteria: (i) continuity of labelling, (ii) resolution of unity, (iii) temporal stability and (iv) action identity [20], that we detail below.

(i) Continuity of labelling refers to the map from the label space $\mathcal{L}$ into Hilbert space. This condition means that the expression ||$\left.z^{\prime}\right\rangle-|z\rangle \mid \rightarrow 0$ whenever $z^{\prime} \rightarrow z$.

(ii) Resolution of unity: a positive measure $\tau(z)$ on $\mathcal{L}$ exists such that the unity operator admits the representation

$$
\int_{\mathcal{L}}|z\rangle\langle z| \mathrm{d} \tau(z)=1
$$


where $|z\rangle\langle z|$ denotes a projector, which takes a state vector into a multiple of the vector $|z\rangle$.

(iii) Temporal stability: the evolution of any coherent state $|z\rangle$ always remains a coherent state, which leads to a relation of the form

$$
|z(t)\rangle=\mathrm{e}^{-\mathrm{i} \hat{H} t / \hbar}|z\rangle
$$

where $z(0)=z$, for all $z \in \mathcal{L}$ and $t$.

(iv) Action identity: this property requires that

$$
\langle z|\hat{H}| z\rangle=|z|^{2} \hbar \omega .
$$

At this point, we remark that requirements (iii) and (iv) are satisfied when the Hamiltonian $\hat{H}$ of the system and its spectrum has the form $E_{n} \sim n \hbar \omega$, where $n$ is the quantum number and $\omega$ is the frequency of the oscillator [20]. In addition, there are some shortcomings about these requirements; for instance, Klauder's states cannot be used for degenerate systems. Furthermore, it is questionable that the action identity can lead to the classical action-angle variable interpretation [21].

\subsection{Applications to Husimi function and Wehrl entropy}

Wehrl's entropy $W$ is a very useful measure of localization in phase space [22]. It is built up using coherent states $[2,19,22]$ and constitutes a powerful tool in statistical physics. The pertinent definition reads

$$
W=-\int \frac{\mathrm{d} x \mathrm{~d} p}{2 \pi \hbar} \mu(x, p) \ln \mu(x, p),
$$

where $\mu(x, p)=\langle z|\hat{\rho}| z\rangle$ is a 'semiclassical' phase-space distribution function associated with the density matrix $\hat{\rho}$ of the system [2,3]. $\mu$ is often referred to as the Husimi distribution [23]. The distribution $\mu(x, p)$ is normalized in the fashion

$$
\int(\mathrm{d} x \mathrm{~d} p / 2 \pi \hbar) \mu(x, p)=1
$$

These last two equations clearly indicate that the Wehrl entropy is simply the 'classical entropy' (6) of a Wigner distribution. Indeed, $\mu(x, p)$ is a Wigner-distribution $D_{W}$ smeared over an $\hbar$-sized region of phase space [19]. The smearing renders $\mu(x, p)$ a positive function, even if $D_{W}$ does not have such a character. The semiclassical Husimi probability distribution refers to a special type of probability: that for simultaneous but approximate location of position and momentum in phase space [19]. The uncertainty principle manifests itself through the inequality $W \geqslant 1$, which was first conjectured by Wehrl [22] and later proved by Lieb [24].

The usual treatment of equilibrium in statistical mechanics makes use of the Gibbs's canonical distribution, whose associated, 'thermal' density matrix is given by

$$
\hat{\rho}=Z^{-1} \mathrm{e}^{-\beta \hat{H}},
$$

with $Z=\operatorname{Tr}\left(\mathrm{e}^{-\beta \hat{H}}\right)$ being the partition function, $\beta=1 / k_{B} T$ the inverse temperature $(T)$ and $k_{B}$ the Boltzmann constant. In order to conveniently write an expression for $W$ consider an arbitrary Hamiltonian $\hat{H}$ of eigenenergies $E_{n}$ and eigenstates $|n\rangle$ ( $n$ stands for a collection of all the pertinent quantum numbers required to label the states). One can always write [19]

$$
\mu(x, p)=\langle z|\hat{\rho}| z\rangle=\frac{1}{Z} \sum_{n} \mathrm{e}^{-\beta E_{n}}|\langle z \mid n\rangle|^{2} .
$$

A useful route to $W$ starts then with equation (9) and continues with equation (6). 


\section{Linear rigid rotor}

We start the present study by exploring a simple model, the linear rigid rotor (LRR), based on the excellent discussion concerning the coherent states for angular momenta given in [25]. The LRR Hamiltonian is [7]

$$
\hat{H}=\frac{\hat{L}^{2}}{2 I_{x y}},
$$

where $\hat{L}^{2}=\hat{L}_{x}^{2}+\hat{L}_{y}^{2}$ is the angular momentum operator and $I_{x}$ and $I_{y}$ are the associated moments of inertia. We have assumed that $I_{x y} \equiv I_{x}=I_{y}$. Calling $|I K\rangle$ the set of $H$ eigenstates, we recall that they verify the relations

$$
\begin{aligned}
& \hat{L}^{2}|I K\rangle=I(I+1) \hbar^{2}|I K\rangle \\
& \hat{L}_{z}|I K\rangle=K \hbar|I K\rangle,
\end{aligned}
$$

with $I=0,1,2 \ldots$, for $-I \leqslant K \leqslant I$, the eigenstates' energy spectrum being given by

$$
\varepsilon_{I}=\frac{I(I+1) \hbar^{2}}{2 I_{x y}} .
$$

Coherent states are constructed in $[4,26]$ for the lineal rigid rotor, using Schwinger's oscillator model of angular momentum, in the fashion

$$
|I K\rangle=\frac{\left(\hat{a}_{+}^{\dagger}\right)^{I+K}\left(\hat{a}_{-}^{\dagger}\right)^{I-K}}{\sqrt{(I+K) !(I-K) !}}|0\rangle,
$$

with $\hat{a}_{+}, \hat{a}_{-}$being the pertinent creation and annihilation operators, respectively, and $|0\rangle \equiv$ $|0,0\rangle$ the vacuum state. The states $|I K\rangle$ are orthogonal and satisfy the closure relation, i.e.,

$$
\begin{aligned}
& \left\langle I^{\prime} K^{\prime} \mid I K\right\rangle=\delta_{I^{\prime}, I} \delta_{K^{\prime}, K}, \\
& \sum_{I=0}^{\infty} \sum_{K=-I}^{I}|I K\rangle\langle I K|=\hat{1} .
\end{aligned}
$$

Since we deal with two degrees of freedom the ensuing coherent states are of the tensor product (denoted by $\otimes$ ) form that involves $\left|z_{1}\right\rangle$ and $\left|z_{2}\right\rangle[25,27]$ :

$$
\left|z_{1} z_{2}\right\rangle=\left|z_{1}\right\rangle \otimes\left|z_{2}\right\rangle
$$

where

$$
\begin{aligned}
& \hat{a}_{+}\left|z_{1} z_{2}\right\rangle=z_{1}\left|z_{1} z_{2}\right\rangle, \\
& \hat{a}_{-}\left|z_{1} z_{2}\right\rangle=z_{2}\left|z_{1} z_{2}\right\rangle .
\end{aligned}
$$

Therefore, the coherent state $\left|z_{1} z_{2}\right\rangle$ writes [25]

$$
\left|z_{1} z_{2}\right\rangle=\mathrm{e}^{-\frac{|z|^{2}}{2}} \mathrm{e}^{z_{1} \hat{a}_{+}^{\dagger}} \mathrm{e}^{z_{2} \hat{a}_{-}^{\dagger}}|0\rangle
$$

with

$$
\begin{aligned}
& \left|z_{1}\right\rangle=\mathrm{e}^{-\frac{\left|z_{1}\right|^{2}}{2}} \mathrm{e}^{z_{1} \hat{a}_{+}^{\dagger}}|0\rangle, \\
& \left|z_{2}\right\rangle=\mathrm{e}^{-\frac{\left|z_{2}\right|^{2}}{2}} \mathrm{e}^{z_{2} \hat{a}_{-}^{\dagger}}|0\rangle .
\end{aligned}
$$


We have introduced the convenient notation

$$
|z|^{2}=\left|z_{1}\right|^{2}+\left|z_{2}\right|^{2}
$$

Using equations (13) and (19) we easily calculate $\left|z_{1} z_{2}\right\rangle$ and, after a bit of algebra, find

$$
\left|z_{1} z_{2}\right\rangle=\mathrm{e}^{-\frac{|z|^{2}}{2}} \sum_{n_{+}, n_{-}} \frac{z_{1}^{n_{+}}}{\sqrt{n_{+} !}} \frac{z_{2}^{n_{-}}}{\sqrt{n_{-} !}}|I K\rangle
$$

where $n_{+}=I+K$ and $n_{-}=I-K$. Therefore, the probability of observing the state $|I K\rangle$ in the coherent state $\left|z_{1} z_{2}\right\rangle$ is of the form

$$
\left|\left\langle I K \mid z_{1} z_{2}\right\rangle\right|^{2}=\mathrm{e}^{-|z|^{2}} \frac{\left|z_{1}\right|^{2 n_{+}}}{n_{+} !} \frac{\left|z_{2}\right|^{2 n_{-}}}{n_{-} !} .
$$

The present coherent states satisfy resolution of unity:

$$
\int \frac{\mathrm{d}^{2} z_{1}}{\pi} \frac{\mathrm{d}^{2} z_{2}}{\pi}\left|z_{1} z_{2}\right\rangle\left\langle z_{1} z_{2}\right|=1 .
$$

Furthermore, $z_{1}$ and $z_{2}$ are continuous variables. We are at this point ready to begin presenting our new results.

\subsection{Husimi distribution}

Following the procedure developed by Anderson et al [19], we can readily calculate the pertinent Husimi distribution [23]. For our system this is defined, from equation (9), as

$$
\mu\left(z_{1}, z_{2}\right)=\left\langle z_{1}, z_{2}|\hat{\rho}| z_{1}, z_{2}\right\rangle
$$

where the density operator is

$$
\hat{\rho}=Z_{2 \mathrm{D}}^{-1} \exp (-\beta \hat{H}) .
$$

Here $\beta$ is the ordinary inverse temperature parameter (this temperature is the ordinary thermodynamic one). The concomitant rotational partition function $Z_{2 \mathrm{D}}$ is given in [7]

$$
Z_{2 \mathrm{D}}=\sum_{I=0}^{\infty}(2 I+1) \mathrm{e}^{-I(I+1) \frac{\Theta}{T}}
$$

with $\Theta=\hbar^{2} /\left(2 I_{x y} k_{B}\right)$. Remark that in the present context speaking of the 'trace operation' entails performing the sum $\operatorname{tr} \equiv \sum_{I=0}^{\infty} \sum_{K=-I}^{I}$. Inserting now the closure relation into equation (26), and using equation (24), we finally get our Husimi distribution in the fashion

$$
\mu\left(z_{1}, z_{2}\right)=\mathrm{e}^{-|z|^{2}} \frac{\sum_{I=0}^{\infty} \frac{|z|^{4 I}}{(2 I) !} \mathrm{e}^{-I(I+1) \frac{\Theta}{T}}}{\sum_{I=0}^{\infty}(2 I+1) \mathrm{e}^{-I(I+1) \frac{\Theta}{T}}} .
$$

It is easy to show that this distribution is normalized to unity

$$
\int \frac{\mathrm{d}^{2} z_{1}}{\pi} \frac{\mathrm{d}^{2} z_{2}}{\pi} \mu\left(z_{1}, z_{2}\right)=1
$$

where $z_{1}$ and $z_{2}$ are given by equations (17), (18) and (22). Note that we must deal with the binomial expression $\left(\left|z_{1}\right|^{2}+\left|z_{2}\right|^{2}\right)^{4 I}$ firstly and then integrate over the whole complex plane (in two dimensions) in order to verify the normalization condition (30). The differential element of area in the $z_{1}\left(z_{2}\right)$ plane is $\mathrm{d}^{2} z_{1}=\mathrm{d} x \mathrm{~d} p_{x} / 2 \hbar\left(\mathrm{d}^{2} z_{2}=\mathrm{d} y \mathrm{~d} p_{y} / 2 \hbar\right)$ [2]. Moreover, we have 


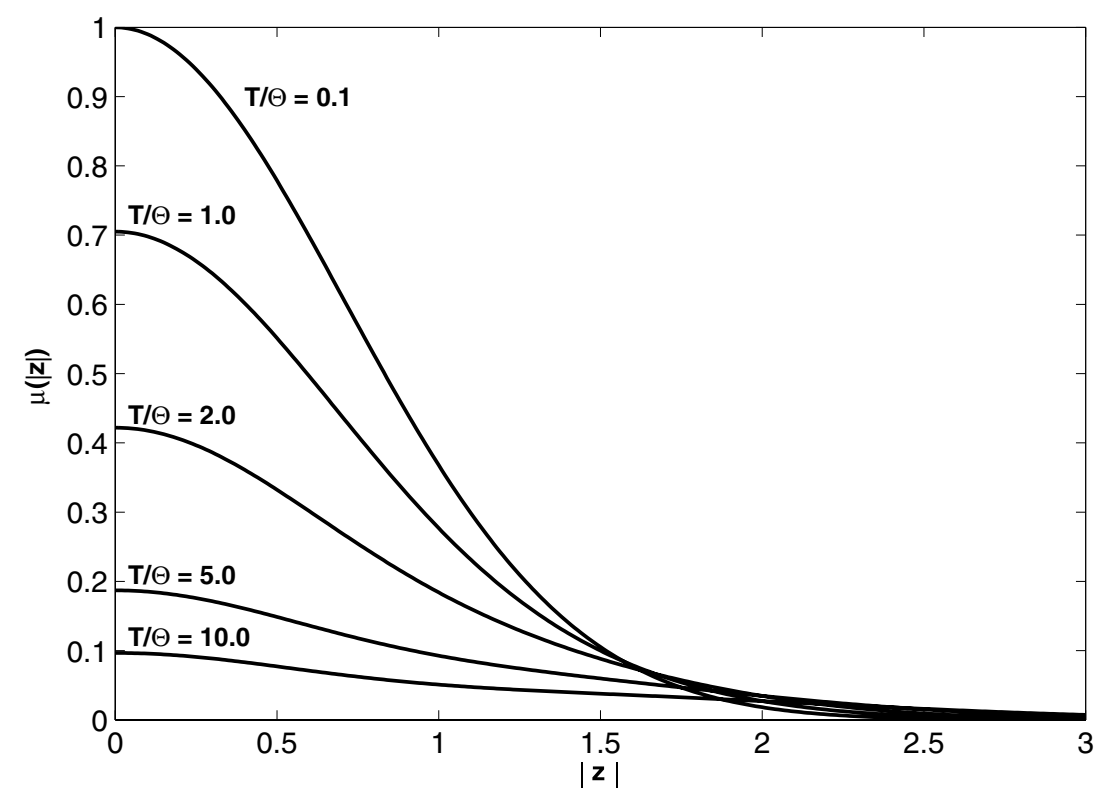

Figure 1. Linear rotor: the Husimi function $\mu\left(z_{1}, z_{2}\right)$ is plotted as a function of $|z|$ for several values of the temperature: from $T / \Theta=0.1$ to $T / \Theta=10$. The behaviour of the Husimi function resembles that of a Gaussian distribution. The peak of the distribution increases as the temperature decreases.

the phase-space relationships

$$
\begin{aligned}
& \left|z_{1}\right|^{2}=\frac{1}{4}\left(\frac{x^{2}}{\sigma_{x}^{2}}+\frac{p_{x}^{2}}{\sigma_{p_{x}}^{2}}\right), \\
& \left|z_{2}\right|^{2}=\frac{1}{4}\left(\frac{y^{2}}{\sigma_{y}^{2}}+\frac{p_{y}^{2}}{\sigma_{p_{y}}^{2}}\right),
\end{aligned}
$$

where $\sigma_{x} \equiv \sigma_{y}=\sqrt{\hbar / 2 m \omega}$ and $\sigma_{p_{x}} \equiv \sigma_{p_{y}}=\sqrt{m \omega \hbar / 2}$. In figure 1, we depict the behaviour of the Husimi distribution $\mu\left(z_{1}, z_{2}\right)$ as a function of $|z|$ at fixed temperature. The profile of the Husimi function is similar to that of a Gaussian distribution.

\subsection{Wehrl entropy and Fisher information}

The Wehrl entropy is a semiclassical measure of localization [22] (so is Fisher's one [12] as well). Indeed, Wehrl's measure is simply a logarithmic Shannon measure built up with Husimi distributions. For the present bi-dimensional model this entropy reads

$$
\mathcal{W}=-\int \frac{\mathrm{d}^{2} z_{1}}{\pi} \frac{\mathrm{d}^{2} z_{2}}{\pi} \mu\left(z_{1}, z_{2}\right) \ln \mu\left(z_{1}, z_{2}\right),
$$

where $\mu\left(z_{1}, z_{2}\right)$ is given by equation (29). The so-called phase-space, shift-invariant Fisher measure [12] is a particular instance of the general Fisher one [10,11] that can be regarded as an (also semiclassical) counterpart of Wehrl entropy [12]. Extending now to the present 
2D case the ideas developed in [12] for the case of the 1D-harmonic oscillator, we define the (phase-space) shift-invariant Fisher measure in the fashion

$$
\mathcal{F}=\int \frac{\mathrm{d}^{2} z_{1}}{\pi} \frac{\mathrm{d}^{2} z_{2}}{\pi} \mu\left(z_{1}, z_{2}\right) \mathcal{A},
$$

with

$$
\begin{aligned}
\mathcal{A}=\sigma_{x}^{2}\left[\frac{\partial \ln \mu\left(z_{1}, z_{2}\right)}{\partial x}\right]^{2}+\sigma_{p_{x}}^{2}\left[\frac{\partial \ln \mu\left(z_{1}, z_{2}\right)}{\partial p_{x}}\right]^{2} \\
+\sigma_{y}^{2}\left[\frac{\partial \ln \mu\left(z_{1}, z_{2}\right)}{\partial y}\right]^{2}+\sigma_{p_{y}}^{2}\left[\frac{\partial \ln \mu\left(z_{1}, z_{2}\right)}{\partial p_{y}}\right]^{2} .
\end{aligned}
$$

It is easy to prove that the quantity $\mathcal{A}$ has the following form, see details in the appendix:

$$
\mathcal{A}=\eta\left(z_{1}, z_{2}\right)^{2}
$$

where

$$
\eta\left(z_{1}, z_{2}\right)=\frac{\sum_{I=0}^{\infty}\left[\frac{|z|^{4 I-1}}{(2 I-1) !}-\frac{|z|^{4 I+1}}{(2 I) !}\right] \mathrm{e}^{-I(I+1) \Theta / T}}{\sum_{I=0}^{\infty} \frac{|z|^{4 I}}{(2 I) !} \mathrm{e}^{-I(I+1) \Theta / T}} .
$$

Therefore, the corresponding Fisher measure acquires the simpler appearance

$$
\mathcal{F}=\int \frac{\mathrm{d}^{2} z_{1}}{\pi} \frac{\mathrm{d}^{2} z_{2}}{\pi} \mu\left(z_{1}, z_{2}\right) \eta\left(z_{1}, z_{2}\right)^{2},
$$

i.e.,

$$
\mathcal{F} \equiv\left\langle\eta\left(z_{1}, z_{2}\right)^{2}\right\rangle,
$$

where with the notation

$$
\langle\mathcal{G}\rangle=\int \frac{\mathrm{d}^{2} z_{1}}{\pi} \frac{\mathrm{d}^{2} z_{2}}{\pi} \mu(z) \mathcal{G},
$$

we allude to the semiclassical expectation value of $\mathcal{G}$.

In figure 2, we plot both Fisher's information and Wehrl's entropy as a function of $T / \Theta$. They behave in different manner. If the temperature $T \rightarrow 0$, Fisher's information measure (inverse delocalization) takes its maximum value and Wehrl's its minimum. This behaviour is reversed for high temperatures, with the degree of delocalization becoming larger and larger. In addition, it compares the rotor results with those for the harmonic oscillator. It depicts, as a function of the temperature, Wehrl's and Fisher's measures in the case of the two models. The overall trend is the same in the two different physical cases, a rather remarkable result.

\subsection{Uncertainty relation}

We calculate now

$$
\left\langle|z|^{n}\right\rangle=\int \frac{\mathrm{d}^{2} z_{1}}{\pi} \frac{\mathrm{d}^{2} z_{2}}{\pi} \mu(z)|z|^{n},
$$

where $n=1,2$ and we find explicitly

$$
\left\langle|z|^{n}\right\rangle=\frac{\sum_{I=0}^{\infty} \frac{\Gamma(2 I+2+n / 2)}{(2 I) !} \mathrm{e}^{-I(I+1) \frac{\Theta}{T}}}{\sum_{I=0}^{\infty}(2 I+1) \mathrm{e}^{-I(I+1) \frac{\Theta}{T}}} .
$$

We see that $\Delta_{z}=\left\langle|z|^{2}\right\rangle-\langle|z|\rangle^{2}$. With this result one can plot the Cramer-Rao product $\mathcal{F} \Delta_{z}$ versus temperature, depicted in figure 3, which indicates how phase-space localization 


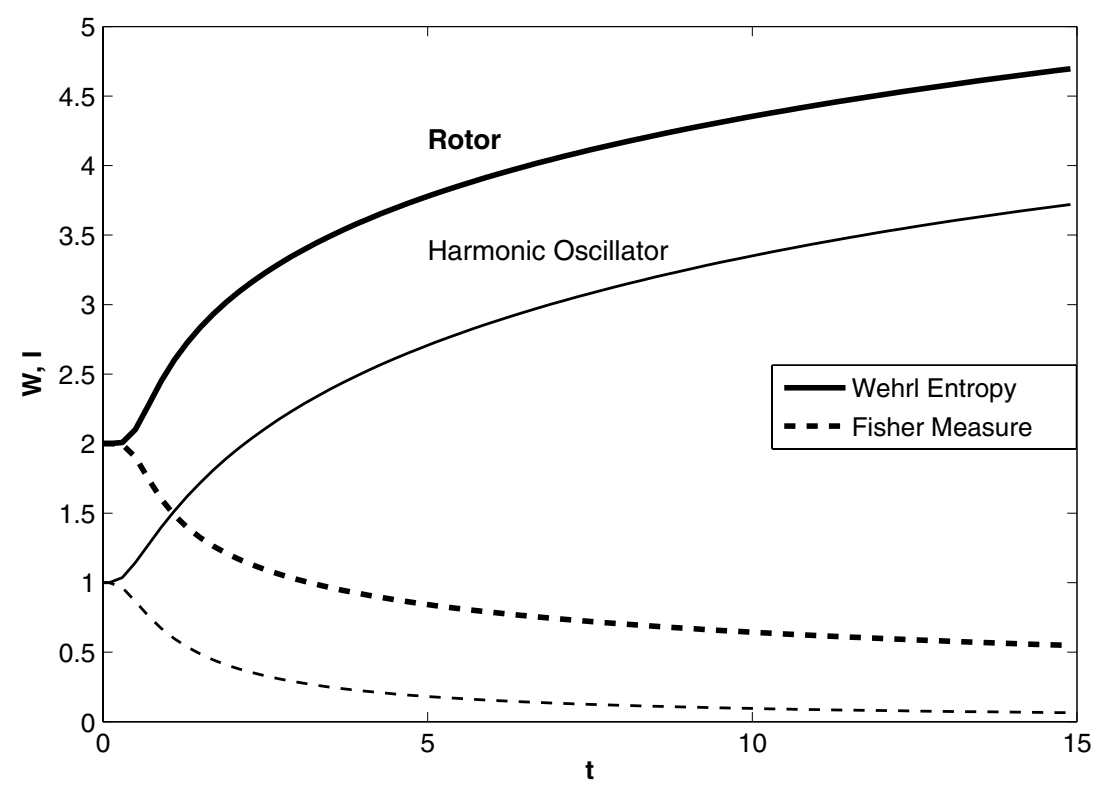

Figure 2. Trends for Fisher's $(\mathcal{F})$ and Wehrl's $(\mathcal{W})$ measures (inverse delocalization) for the linear rotor in comparison with those for the harmonic oscillator. The horizontal axis is the temperature. If it increases, the Fisher information decreases while Wehrl entropy increases. It must be noted that, in the case of harmonic oscillator, $t=k T / \hbar \omega$, and in the case of rotor, $t=k T\left(2 I_{x y}\right) / \hbar^{2}$.

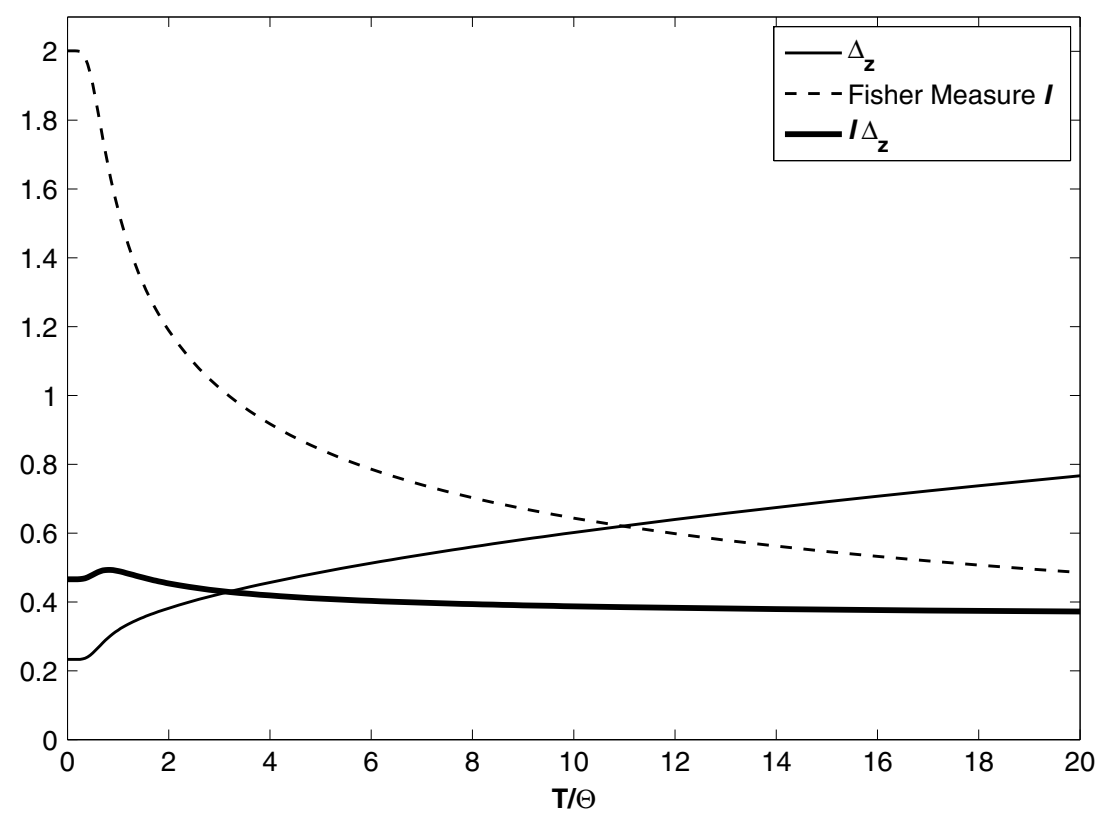

Figure 3. Trends for Fisher's $(\mathcal{F})$ measure (inverse delocalization), $\Delta_{z}=\left\langle|z|^{2}\right\rangle-\langle|z|\rangle^{2}$ and the Cramer-Rao product $\mathcal{F} \Delta_{z}$ as a function of the temperature for the linear rotor. The localization information is approximately constant over a wide temperature range. 
ability changes with temperature. The strange behaviour near $T=0$ should not cause undue worries because the semiclassical approximation is not valid near $T=0$. Curiously enough, our localization information remains relatively constant over a wide temperature range. The inescapable conclusion is that the Cramer-Rao localization estimator is not affected by temperature. This independence from $T$ makes the Cramer-Rao estimator a much better one than Wehrl's entropy that strongly depends on the temperature.

\section{Rigid rotor in three dimensions}

In the present section we consider a more general problem, the 3D-rigid rotor model, whose Hamiltonian writes [1]

$$
\hat{H}=\frac{\hat{L}_{x}^{2}}{2 I_{x}}+\frac{\hat{L}_{y}^{2}}{2 I_{y}}+\frac{\hat{L}_{z}^{2}}{2 I_{z}}
$$

where $I_{x}, I_{y}$ and $I_{z}$ are the associated moments of inertia. A complete set of rotor eigenstates is $\{|I M K\rangle\}$. The following relations apply:

$$
\begin{aligned}
\hat{L}^{2}|I M K\rangle & =I(I+1) \hbar^{2}|I M K\rangle \\
\hat{L}_{z}|I M K\rangle & =K \hbar|I M K\rangle \\
\hat{J}_{z}|I M K\rangle & =M \hbar|I M K\rangle,
\end{aligned}
$$

where $I=0, \ldots, \infty,-I \leqslant K \leqslant I$ and $-I \leqslant M \leqslant I$. The states $|I M K\rangle$ satisfy orthogonality and closure relation [1]:

$$
\begin{aligned}
& \left\langle I^{\prime} M^{\prime} K^{\prime} \mid I M K\right\rangle=\delta_{I^{\prime}, I} \delta_{M^{\prime}, M} \delta_{K^{\prime}, K}, \\
& \sum_{I=0}^{\infty} \sum_{M=-I}^{I} \sum_{K=-I}^{I}|I M K\rangle\langle I M K|=\hat{1}
\end{aligned}
$$

If we take $\hat{L}^{2}=\hat{L}_{x}^{2}+\hat{L}_{y}^{2}+\hat{L}_{z}^{2}$ and assume axial symmetry, i.e., $I_{x y} \equiv I_{x}=I_{y}$, we can recast the Hamiltonian as

$$
\hat{H}=\frac{1}{2 I_{x y}}\left[\hat{L}^{2}+\left(\frac{I_{x y}}{I_{z}}-1\right) \hat{L}_{z}^{2}\right],
$$

where $\hat{L}^{2}$ is the angular momentum operator and $\hat{L}_{z}$ is its projection on the rotation axis $z$. The concomitant spectrum of energy becomes

$$
\varepsilon_{I, K}=\frac{\hbar^{2}}{2 I_{x y}}\left[I(I+1)+\left(\frac{I_{x y}}{I_{z}}-1\right) K^{2}\right],
$$

where $I=0,1,2, \ldots$ and it represents the eigenvalue of the angular momentum operator $\hat{L}^{2}$, the numbers $m=-I, \ldots,-1,0,1, \ldots, I$ stand for the projections on the intrinsic rotation axis of the rotor. All states exhibit a $(2 I+1)$ degeneracy. The parameters $I_{x}=I_{y} \equiv I_{x y}$ and $I_{z}$ are the inertia momenta. Different 'geometrical' instances are characterized through the $I_{x y} / I_{z}$ ratio. For example, the value $I_{x y} / I_{z}=1$ corresponds to the spherical rotor. Limiting cases can also be considered. This is, $I_{x y} / I_{z}=1 / 2$ and $I_{x y} / I_{z} \rightarrow \infty$, that correspond to the extremely oblate and prolate cases, respectively. 


\subsection{Coherent states for the rigid rotor in three dimensions}

In order to obtain the Husimi distribution for this problem we need first of all to have the associated coherent states. Morales et al have constructed them in [1] and discussed their mathematical foundations. First, they introduced the auxiliary quantity

$$
X_{I, M, K}=\sqrt{I !(I+M) !(I-M) !(I+K) !(I-K) !},
$$

and then write [1]

$$
\left|z_{1} z_{2} z_{3}\right\rangle=\mathrm{e}^{-\frac{|u|^{2}}{2}} \sum_{I M K} \frac{[(2 I) !]^{2} z_{1}^{(I+M)} z_{2}^{I} z_{3}^{(I+K)}}{X_{I, M, K}}|I M K\rangle,
$$

where the following supplementary variable were introduced by Morales et al in [1]:

$$
|u|^{2}=\left|z_{2}\right|^{2}\left(1+\left|z_{1}\right|^{2}\right)^{2}\left(1+\left|z_{3}\right|^{2}\right)^{2} .
$$

All coherent states share at least two requirements. Continuity of labelling and resolution of unity. In relation to the last property we add

$$
\int \mathrm{d} \Gamma\left|z_{1} z_{2} z_{3}\right\rangle\left\langle z_{1} z_{2} z_{3}\right|=1
$$

where $\mathrm{d} \Gamma$ is the measure of integration given by [1]

$\mathrm{d} \Gamma=\mathrm{d} \tau\left\{4\left[\left(1+\left|z_{1}\right|^{2}\right)\left(1+\left|z_{3}\right|^{2}\right)\right]^{4}\left|z_{2}\right|^{4}-8\left[\left(1+\left|z_{1}\right|^{2}\right)\left(1+\left|z_{3}\right|^{2}\right)\right]^{2}\left|z_{2}\right|^{2}+1\right\}$

with

$$
\mathrm{d} \tau=\frac{\mathrm{d}^{2} z_{1}}{\pi} \frac{\mathrm{d}^{2} z_{2}}{\pi} \frac{\mathrm{d}^{2} z_{3}}{\pi}
$$

and, of course, in this case we have three degrees of freedom. The present formulation satisfies the weaker version of the second requirement, because the measure is defined nonpositive [1].

\subsection{Husimi function, Wehrl entropy and Fisher measure}

Using now equation (50) we find

$$
\left|\left\langle I M K \mid z_{1} z_{2} z_{3}\right\rangle\right|^{2}=\frac{\mathrm{e}^{-|u|^{2}}}{X_{I, M, K}^{2}}[(2 I) !]^{2}\left|z_{1}\right|^{2(I+M)}\left|z_{2}\right|^{2 I}\left|z_{3}\right|^{2(I+K)}
$$

and determine that, in this case, the rotational partition function reads

$$
Z_{3 \mathrm{D}}=\sum_{I=0}^{\infty} \sum_{K=-I}^{I} \sum_{M=-I}^{I} \mathrm{e}^{-\beta \varepsilon_{I, K}},
$$

i.e.,

$$
Z_{3 \mathrm{D}}=\sum_{I=0}^{\infty}(2 I+1) \mathrm{e}^{-I(I+1) \frac{\Theta}{T}} \sum_{K=-I}^{I} \exp \left(-\left(\frac{I_{x y}}{I_{z}}-1\right) K^{2} \frac{\Theta}{T}\right) .
$$

Remark that if we take the 'extremely prolate' limiting case $I_{x y} / I_{z} \rightarrow \infty$ just one term survives in the right sum of the right-hand side in equation (57), that for $K=0$, while all terms for $K \neq 0$ vanish. In this special instance case $Z_{2 \mathrm{D}}$ is recovered from $Z_{3 \mathrm{D}}$. The pertinent Husimi distribution becomes

$$
\mu\left(z_{1}, z_{2}, z_{3}\right)=\frac{\mathrm{e}^{-|u|^{2}}}{Z_{3 \mathrm{D}}} \sum_{I=0}^{\infty} \frac{(2 I) !}{I !}|v|^{2 I} \mathrm{e}^{-I(I+1) \frac{\Theta}{T}} \times g(I),
$$


where

$$
g(I)=\sum_{K=-I}^{I} \frac{\left|z_{3}\right|^{2(I+K)}}{(I+K) !(I-K) !} \exp \left(-\left(\frac{I_{x y}}{I_{z}}-1\right) K^{2} \frac{\Theta}{T}\right)
$$

with

$$
\begin{aligned}
& |v|^{2}=\left(1+\left|z_{1}\right|^{2}\right)^{2}\left|z_{2}\right|^{2}, \\
& |u|^{2}=|v|^{2}\left(1+\left|z_{3}\right|^{2}\right)^{2} .
\end{aligned}
$$

We can easily verify that $\mu\left(z_{1}, z_{2}, z_{3}\right)$ is normalized in the fashion

$$
\int \mathrm{d} \Gamma \mu\left(z_{1}, z_{2}, z_{3}\right)=1
$$

We compute now (i) the Wehrl entropy in the form

$$
\mathcal{W}=\int \mathrm{d} \Gamma \mu\left(z_{1}, z_{2}, z_{3}\right) \ln \mu\left(z_{1}, z_{2}, z_{3}\right),
$$

and (ii) the Fisher measure as follows:

$$
\mathcal{F}=\int \mathrm{d} \Gamma \mu\left(z_{1}, z_{2}, z_{3}\right) \mathcal{A}_{3 \mathrm{D}}
$$

where in this case we define the three-dimensional quantity $\mathcal{A}_{3 \mathrm{D}}$ in the fashion

$$
\begin{aligned}
\mathcal{A}_{3 \mathrm{D}}=\sigma_{x}^{2}\left[\frac{\partial \ln \mu\left(z_{1}, z_{2}\right)}{\partial x}\right]^{2}+\sigma_{p_{x}}^{2}\left[\frac{\partial \ln \mu\left(z_{1}, z_{2}\right)}{\partial p_{x}}\right]^{2}+\sigma_{y}^{2}\left[\frac{\partial \ln \mu\left(z_{1}, z_{2}\right)}{\partial y}\right]^{2} \\
+\sigma_{p_{y}}^{2}\left[\frac{\partial \ln \mu\left(z_{1}, z_{2}\right)}{\partial p_{y}}\right]^{2}+\sigma_{z}^{2}\left[\frac{\partial \ln \mu\left(z_{1}, z_{2}\right)}{\partial z}\right]^{2}+\sigma_{p_{z}}^{2}\left[\frac{\partial \ln \mu\left(z_{1}, z_{2}\right)}{\partial p_{z}}\right]^{2},
\end{aligned}
$$

with the phase-space relationships (31), (32) and

$$
\left|z_{3}\right|^{2}=\frac{1}{4}\left(\frac{z^{2}}{\sigma_{z}^{2}}+\frac{p_{z}^{2}}{\sigma_{p_{z}}^{2}}\right)
$$

where $\sigma_{z}=\sqrt{\hbar / 2 m \omega}$ and $\sigma_{p_{z}}=\sqrt{m \hbar \omega / 2}$. In this instance $\mathrm{d}^{2} z_{3}=\mathrm{d} z \mathrm{~d} p_{z} / 2 \hbar$. Finally, we are led to

$$
\mathcal{F}=\int \mathrm{d} \Gamma \mu\left(z_{1}, z_{2}, z_{3}\right)\left\{\gamma^{2}\left(\left|z_{1}\right|^{2}\left|z_{2}\right|^{2}+\frac{1}{4}\left(1+\left|z_{1}\right|^{2}\right)^{2}\right)+4|u|^{2}\left|z_{3}\right|^{2}\right\},
$$

i.e.,

$$
\mathcal{F}=\left\langle\gamma^{2}\left(\left|z_{1}\right|^{2}\left|z_{2}\right|^{2}+\frac{1}{4}\left(1+\left|z_{1}\right|^{2}\right)^{2}\right)+4|u|^{2}\left|z_{3}\right|^{2}\right\rangle,
$$

where

$$
\begin{array}{r}
\gamma=\frac{-\left(1+\left|z_{3}\right|^{2}\right)^{2} \sum_{I=0}^{\infty} \frac{2(2 I) !}{I !}|v|^{2 I+1} \mathrm{e}^{-I(I+1) \frac{\Theta}{T}} \times g(I)}{\sum_{I=0}^{\infty} \frac{(2 I) !}{I !}|v|^{I I} \mathrm{e}^{-I(I+1) \frac{\Theta}{T}} \times g(I)} \\
+\frac{\sum_{I=0}^{\infty} \frac{2(2 I) !}{(I-1) !}|v|^{2 I-1} \mathrm{e}^{-I(I+1) \frac{\Theta}{T}} \times g(I)}{\sum_{I=0}^{\infty} \frac{(2 I) !}{I !}|v|^{2 I} \mathrm{e}^{-I(I+1) \frac{\Theta}{T}} \times g(I)} .
\end{array}
$$




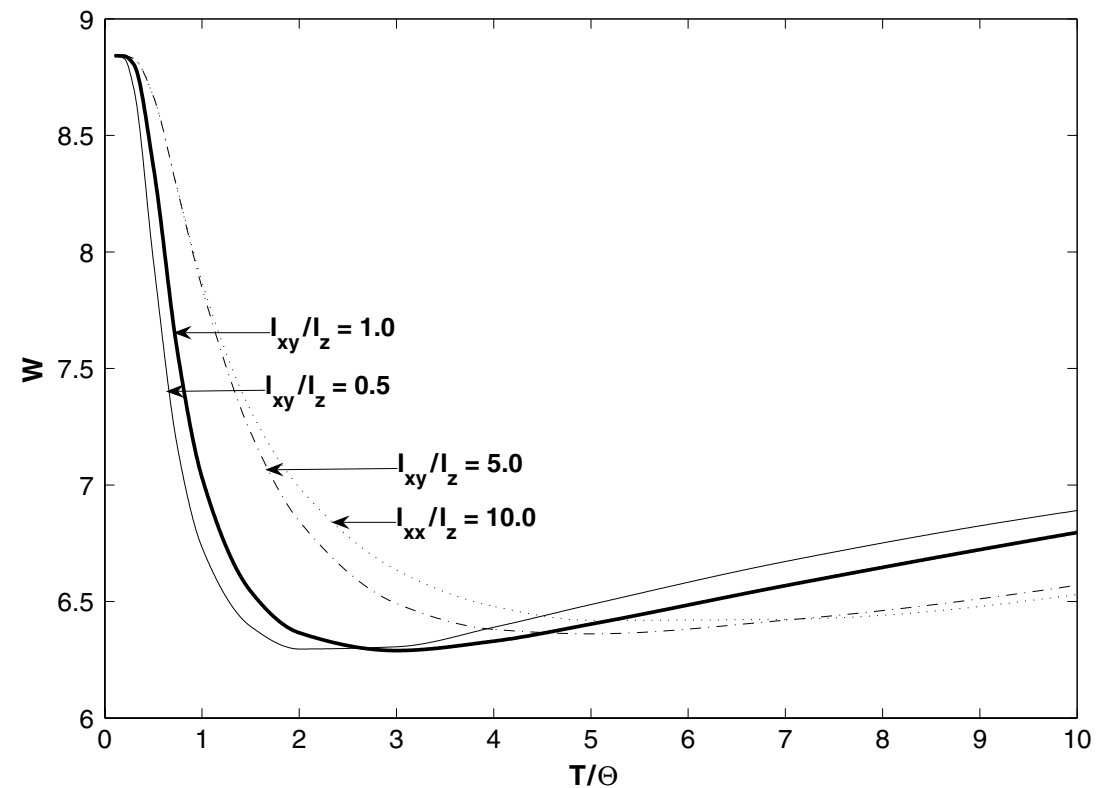

Figure 4. 3D-anisotropic rotor. We plot Wehrl's entropy $\mathcal{W}$ as a function of $T / \Theta$ for several values of the anisotropy ratio $\left(I_{x y} / I_{z}=1 / 2,1,5,10\right)$. Trends for the Wehrl entropy are not quite similar to those for the linear case. A maximum is seen at $T / \Theta=0$ and a minimum at $T / \Theta \geqslant 2$, whose exact values depend on the ratio $I_{x y} / I_{z}$.

In the special instance $I_{x y} / I_{z}=1$, that corresponds to the spherical rotor, we explicitly obtain

$$
\mu\left(z_{1}, z_{2}, z_{3}\right)=\mathrm{e}^{-|u|^{2}} \frac{\sum_{I=0}^{\infty} \frac{|u|^{2 I}}{I !} \mathrm{e}^{-I(I+1) \frac{\Theta}{T}}}{\sum_{I=0}^{\infty}(2 I+1)^{2} \mathrm{e}^{-I(I+1) \frac{\Theta}{T}}} .
$$

Having the Husimi functions the Wehrl entropy is straightforwardly computed.

In order to emphasize some special cases associated with possible applications we consider several possibilities.

(i) The spherical rotor $I_{x y}=I_{x}=I_{y}=I_{z}$, thus $I_{x y} / I_{z}=1$ (e.g. $\left.\mathrm{CH}_{4}\right)$.

(ii) The oblate rotor $I_{x y}=I_{x}=I_{y}<I_{z}$, specifically $1 / 2 \leqslant I_{x y} / I_{z}<1$ (e.g. $\left.\mathrm{C}_{6} \mathrm{H}_{6}\right)$.

(iii) The prolate rotor $I_{x y}=I_{x}=I_{y}>I_{z}$, which corresponds to $I_{x y} / I_{z}>1$ (e.g. $\mathrm{PCl}_{5}$ ).

(iv) The extremely prolate rotor is equivalent to the linear case (all diatomic molecules) $I_{z}=0$, this is $I_{x y} / I_{z} \rightarrow \infty$ (e.g. $\mathrm{CO}_{2}, \mathrm{C}_{2} \mathrm{H}_{2}$ ).

In figure 4 , we depict the Wehrl entropy $\mathcal{W}$, as a function of $T / \Theta$, for several values of $I_{x y} / I_{z}$. This is the extremely oblate rotor $I_{x y} / I_{z}=1 / 2$, the prolate rotor $I_{x y} / I_{z}=5,10$ and the spherical case $I_{x y} / I_{z}=1$.

\section{Concluding remarks}

A study has been performed on the basis of Schwinger's angular momenta construction [4] and following Morales et al [1] coherent states' rotor formulation. Possible applications to specific molecular problems have been suggested. 
We have concentrated our efforts on the study of the semiclassical behaviour of the rigid rotor and have obtained in analytical fashion the form of the appropriate semiclassical Husimi distribution for two cases, namely, the linear and the axially symmetric rigid rotor. The linear case can be reobtained as a particular instance of the three-dimensional formulation. We have also obtained an analytical expression of the shift-invariant Fisher measure built up with Husimi distributions for the rigid rotor model, concluding that Fisher's measure is a better indicator of the delocalization than Wehrl's entropy.

\section{Acknowledgments}

S Curilef and F Pennini would like to thank partial financial support by FONDECYT 1051075 and DGIP-UCN 2006.

\section{Appendix}

First of all, we carry out the differentiation of the Husimi distribution (29) with respect to the variable $x$, obtaining the following result:

$$
\frac{\partial \ln \mu\left(z_{1}, z_{2}\right)}{\partial x}=2 \eta\left(z_{1}, z_{2}\right) \frac{\partial|z|}{\partial x},
$$

where the quantity $\eta\left(z_{1}, z_{2}\right)$ was defined in equation (37). Moreover, from equations (31) and (32) we have

$$
\frac{\partial|z|}{\partial x}=\frac{x}{4|z| \sigma_{x}^{2}}
$$

and we are led to

$$
\frac{\partial \ln \mu\left(z_{1}, z_{2}\right)}{\partial x}=\frac{\eta\left(z_{1}, z_{2}\right) x}{2|z| \sigma_{x}^{2}} .
$$

We arrive at a similar expression differentiating with respect to $p_{x}$,

$$
\frac{\partial \ln \mu\left(z_{1}, z_{2}\right)}{\partial p_{x}}=\frac{\eta\left(z_{1}, z_{2}\right) p_{x}}{2|z| \sigma_{p_{x}}^{2}} .
$$

Analogous expressions are obtained by replacing $x$ and $p_{x}$ with $y$ and $p_{y}$, respectively. Finally, substituting these results into equation (35) we thus arrive at

$$
\mathcal{A}=\eta\left(z_{1}, z_{2}\right)^{2} \text {. }
$$

\section{References}

[1] Morales J A, Deumens E and Öhrn Y 1999 J. Math. Phys. 40776

[2] Glauber R J 1963 Phys. Rev. 1312766

[3] Klauder J R and Skagerstam B S 1985 Coherent States (Singapore: World Scientific)

[4] Sakurai J J 1994 Modern Quantum Mechanics ed S F Tuan (Reading, MA: Addison-Wesley) p 217

[5] Casida M E, Harriman J E and Anchell J L 1987 Int. J. Quantum Chem. 32435

[6] Harriman J E and Casida M E 1993 Int. J. Quantum Chem. 45263

[7] Pathria R K 1993 Statistical Mechanics (Exeter: Pergamon)

[8] Garbaczewski P 2005 Entropy 7253

[9] Frieden B R and Soffer B H 1995 Phys. Rev. E 522274

[10] Frieden B R 1998 Physics from Fisher Information (Cambridge: Cambridge University Press)

[11] Frieden B R 2003 Science from Fisher Information (Cambridge: Cambridge University Press)

[12] Pennini F and Plastino A 2004 Phys. Rev. E 69057101 
[13] Ullah N 1994 Phys. Rev. E 491743

[14] Arranz F J, Borondo F and Benito R M 1996 Phys. Rev. E 542458

[15] Titantah J T and Hounkonnou M N 1999 J. Phys. A: Math. Gen. 32897

[16] Titantah J T and Hounkonnou M N 1997 J. Phys. A: Math. Gen. 306327

[17] Titantah J T and Hounkonnou M N 1997 J. Phys. A: Math. Gen. 306347

[18] Titantah J T and Hounkonnou M N 1995 J. Phys. A: Math. Gen. 286345

[19] Anderson A and Halliwell J J 1993 Phys. Rev. D 482753

[20] Gazeau J P and Klauder J R 1999 J. Phys. A: Math Gen. 32123

[21] Thaik M and Inomata A 2005 J. Phys. A: Math Gen. 381767

[22] Wehrl A 1979 Rep. Math. Phys. 16353

[23] Husimi K 1940 Proc. Phys. Math. Soc. Japan 22264

[24] Lieb E H 1978 Commun. Math. Phys. 6235

[25] Nieto M M 1980 Phys. Rev. D 22391

[26] Schwinger J 1965 In Quantum Theory of Angular Momentum ed L C Biedenharn and H van Dam (New York: Academic) p 229

[27] Cohen-Tannoudji C, Diu B and Laloë F 1977 Quantum Mechanics (Paris: Hermann) 